\title{
GAUSSIAN WIGNER DISTRIBUTIONS: A COMPLETE CHARACTERIZATION
}

\author{
R. SIMON \\ The Institute of Mathematical Sciences, CITCampus, Madras 600113, India
}

E.C.G. SUDARSHAN

Centrefor Particle Theory, The University of Texas atAustin, Austin, TX 78712, USA

and

N. MUKUNDA

Centre for Theoretical Studies, Indian Institute of Science, Bangalore 560012, India

\begin{abstract}
Necessary and sufficient conditions on a gaussian phase space distribution to be a bona fide Wigner distribution, for any number of degrees of freedom, are derived. Williamson's theorem on the normal forms of positive matrices under symplectic transformations is used in a fundamental way.
\end{abstract}

The following question arises in the analysis of problems of interest in both quantum mechanics and optics: if a hermitian operator $t$ acting on the Hilbert space $\mathscr{H}=\mathrm{L}^{2}\left(\mathbb{R}^{n}\right)$ has a configuration space kernel of the gaussian form:

$$
\begin{aligned}
& \left\langle\boldsymbol{q}|\hat{\digamma}| \boldsymbol{q}^{\prime}\right\rangle \equiv \Gamma\left(\boldsymbol{q} ; \boldsymbol{q}^{\prime}\right) \\
& \quad=(2 / \pi)^{n / 2}(\operatorname{det} L)^{1 / 2} \exp \left[-\boldsymbol{q}^{\mathrm{T}} L \boldsymbol{q}-\boldsymbol{q}^{\mathrm{T}} L \boldsymbol{q}-\frac{1}{2}\left(\boldsymbol{q}-\boldsymbol{q}^{\prime}\right)^{\mathrm{T}} M\left(\boldsymbol{q}-\boldsymbol{q}^{\prime}\right)+\frac{1}{2} \mathrm{i}\left(\boldsymbol{q}-\boldsymbol{q}^{\prime}\right)^{\mathrm{T}} K\left(\boldsymbol{q}+\boldsymbol{q}^{\prime}\right)\right],
\end{aligned}
$$

what are the necessary and sufficient conditions on $L, M$ and $K$ to ensure that $\hat{\Gamma}$ is positive semidefinite? Here $q$ and $q^{\prime}$ are $n$-component column vectors; $L$ and $M$ are real symmetric $n \times n$ matrices, while $K$ is a real $n \times n$ matrix. It is easy to see that the expression in the exponent is the most general one quadratic in $q$ and $\boldsymbol{q}^{\prime}$, and invariant under complex conjugation followed by interchange of $q$ and $\boldsymbol{q}^{\prime}$. The number of independent real parameters in this family of gaussian kernels is $n(2 n+1)$, the same as the number of parameters in the group $\operatorname{Sp}(2 n, \mathrm{R})$.

In quantum mechanics, the density matrix of an $n$-dimensional oscillator system in thermal equilibrium and its transforms under unitary $\operatorname{Sp}(2 n, \mathbb{R})$ action, has the above gaussian form. In optics, the cross-spectral density of gaussian Schell-model fields [1,2], which have attracted great interest in recent studies on radiometry of partially coherent sources [3], have precisely this form.

The purpose of this letter is to answer the question posed in the opening paragraph. For this it is useful to compute the Moyal transform $W(q ; \boldsymbol{p})$ of the kernel $(1)$. The necessary and sufficient condition for its existence is that $L+M$ be positive definite, and then [4] 


$$
\begin{aligned}
& W(\boldsymbol{q} ; \boldsymbol{p}) \equiv(2 \pi)^{-n} \int_{\mathbb{Q}^{n}} \mathrm{~d}^{n} \boldsymbol{q}^{\prime} \Gamma\left(\boldsymbol{q}-\frac{1}{2} \boldsymbol{q}^{\prime} ; \boldsymbol{q}+\frac{1}{2} \boldsymbol{q}^{\prime}\right) \exp \left(\boldsymbol{i p}^{\mathrm{T}} \boldsymbol{q}^{\prime}\right) \\
& =\pi^{-n}[\operatorname{det} L / \operatorname{det}(L+M)]^{1 / 2} \exp \left[-2 \boldsymbol{q}^{\mathrm{T}} L \boldsymbol{q}-\frac{1}{2}(\boldsymbol{p}-K \boldsymbol{q})^{\mathrm{T}}(L+M)^{-1}(\boldsymbol{p}-K \boldsymbol{q})\right] .
\end{aligned}
$$

$\Gamma\left(\boldsymbol{q} ; \boldsymbol{q}^{\prime}\right)$ being a bona fide density matrix (cross-spectral density) corresponds to $W(\boldsymbol{q} ; \boldsymbol{p})$ being a Wigner distribution (Wolf function) [5,6]. $W(\boldsymbol{q} ; \boldsymbol{p})$ is more conveniently expressed by arranging $q$ and $\boldsymbol{p}$ into a $2 n$-component real column vector $Q$ :

$$
Q=0
$$

and defining a real symmetric $2 n \times 2 n$ matrix $G$ :

$$
G=\left(\begin{array}{cc}
A & C \\
C^{\mathrm{T}} & B
\end{array}\right), \quad A=2 L+\frac{1}{2} K^{\mathrm{T}}(L+M)^{-1} K, \quad B=\frac{1}{2}(L+M)^{-1}, \quad C=-\frac{1}{2} K^{\mathrm{T}}(L+M)^{-1} .
$$

Given the properties of $L, M$ and $K$, it can be seen that there are no algebraic relations on the elements of $G$ beyond symmetry. The Moyal transform now has the compact gaussian form

$$
W(\boldsymbol{q} ; \boldsymbol{p})=W(Q)=\pi^{-n}(\operatorname{det} G)^{1 / 2} \exp \left(-Q^{\mathrm{T}} G Q\right) .
$$

Eqs. (4) can be inverted to express $L, M, K$ in terms of $A, B, C$, consistent with the invertibility of the Moyal transform. Our question can now be phrased in this form: What are the necessary and sufficient conditions on the parameter matrix $G$ so that the gaussian phase-space distribution (5) will be a Wigner distribution? \#1

A density matrix must be hermitian, have unit trace, and be positive semidefinite. Hermiticity of $\hat{F}$ has been ensured through reality of $L, M$ and $K$. The kernel (1) is traceable if and only if $L$ is positive definite, $L>0$; then our normalisation factor ensures that $\hat{T}$ has unit trace. It is the positive semidefiniteness property which is somewhat subtle, so we implement it in two stages. First we derive a necessary condition. Positive semidefmiteness of $\hat{\Gamma}$ implies the existence of a unique hermitian positive semidefmite square $\operatorname{root} \hat{\Gamma}^{1 / 2}$. Applying the Schwarz inequality to the vectors $\hat{\Gamma}^{1 / 2}|\boldsymbol{q}\rangle, \hat{\Gamma}^{1 / 2}\left|\boldsymbol{q}^{\prime}\right\rangle$ gives

$$
\left|\Gamma\left(\boldsymbol{q} ; \boldsymbol{q}^{\prime}\right)\right|^{2} \leqslant \Gamma(\boldsymbol{q} ; \boldsymbol{q}) \Gamma\left(\boldsymbol{q}^{\prime} ; \boldsymbol{q}^{\prime}\right)
$$

which for the gaussian kernel (1) reads

$$
\exp \left[-\left(\boldsymbol{q}-\boldsymbol{q}^{\prime}\right) M\left(\boldsymbol{q}-\boldsymbol{q}^{\prime}\right) \leqslant 1 \text { for all } q, \boldsymbol{q}^{\prime},\right.
$$

i.e.,

$$
M \geqslant 0 .
$$

The conditions on $L$ and $M$ so far are $L>0, M \geqslant 0, L+M>0$, with the first two clearly ensuring the third. If we write $G$ in the factored form

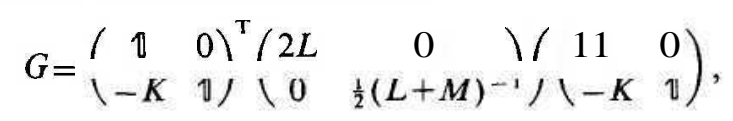

we see that $L>0, L+M>0$ imply, and are implied by, $G$ being positive definite. Thus a necessary condition for $\hat{F}$ to be a density matrix or $W(Q)$ a Wigner distribution is

$$
\mathrm{G}>0 \text {. }
$$

\# See ref. [7], and in particular the discussion following eq. (8.15) therein. 
To arrive at a set of sufficient conditions we analyse the behaviour of $f$ under unitary $\operatorname{Sp}(2 n, \mathrm{R})$ action. The defining representation of this group consists of real $2 n \times 2 n$ matrices $S$ obeying

$$
S^{\mathrm{T}} \beta S=\beta, \quad \beta=\mathrm{i} \sigma_{2} \times 1_{n \times n}=\left(\begin{array}{rr}
0 & 1 \\
-1 & 0
\end{array}\right) .
$$

This form of $f t$ corresponds to arranging all the $q$ first, and then all the $p$, in the column vector $Q$ in eq. (3). $\mathrm{Sp}(2 n, \mathrm{R})$ is the group of linear transformations which leave invariant the fundamental commutators among $n$ canonical pairs of operators. The generators in the defining representation are of the form $f t G$ where the $G$ are real symmetric matrices. Along with $S$, both $S^{-1}$ and $S^{\mathrm{T}}$ also belong to $\mathrm{Sp}(2 n, \mathrm{R})$. The unitary action of $\operatorname{Sp}(2 n, \mathrm{R})$ on $\mathscr{H}$ is via operators $U(S)$ whose generators are symmetric hermitian quadratic expressions in the canonical operators, and on $f$ the action is by conjugation:

$$
S: \hat{\Gamma} \rightarrow \hat{\Gamma}=U(S) \hat{\Gamma} U(S)^{-1} .
$$

We see that the defining properties of density matrices are $\operatorname{Sp}(2 n, \mathrm{R})$ invariant. In particular, if $f$ is positive semidefinite, so is $f^{\prime}$. The change in the Moyal transform accompanying (11) is easier to handle:

$$
S: W(Q) \rightarrow W^{\prime}(Q)=W\left(S^{-1} Q\right) .
$$

For the gaussian expression (5) this means a symmetric transformation on $G$ and a similarity transformation on $\beta G$ :

$$
G \rightarrow G^{\prime}=\left(S^{-1}\right)^{\mathrm{T}} G S^{-1}, \quad \beta C ?^{\prime}=S \beta G S^{-1} .
$$

Condition (9) on $\mathrm{G}$ is clearly preserved by $\mathrm{Sp}(2 n, \mathrm{R})$.

Now we make use of a fundamental theorem due to Williamson [8]. It states that the normal form $G_{0}$ of a real symmetric positive definite matrix $G$ under the $\operatorname{Sp}(2 n, \mathbb{R})$ transformation (13) is diagonal with positive elements. The normal form can be written as

$$
G_{0}=\left(\begin{array}{llllllll}
K_{1} & & & & & & \\
& K_{2} & & & & 0 & & \\
& & \ddots & & & & & \\
& & & K_{n} & & & & \\
& & & & K_{1} & & & \\
& & 0 & & & K_{2} & & \\
& & & & & & \ddots & \\
& & & & & & K_{n}
\end{array}\right) \text {, }
$$

where $\left(G_{0}\right)_{n+m, n+m}=\left(G_{0}\right)_{m, m}$ for $m=l, 2, \ldots, n$ has been achieved by using the fact that scaling each $q_{m}$ by a positive factor $\gamma_{m}$ and each $p_{m}$ by the reciprocal $\gamma_{m}^{-1}$ is an $\operatorname{Sp}(2 n, \mathrm{R})$ transformation. Given $f$ with parameter matrix $G$, it is positive semidefinite if and only if $\hat{\digamma}_{0}$ corresponding to the normal form $G_{0}$ is positive semidefinite. In the normal form this property is easily tested.

For the matrix $G_{0}$ of (14), $K_{0}=0$ while

$$
A_{0}=B_{0}=\left(\begin{array}{llll}
K_{1} & & & 0 \\
& K_{2} & & \\
& & \ddots & \\
0 & & & K_{n}
\end{array}\right),
$$

so that

$$
M_{0}=\frac{1}{2} \operatorname{diag}\left(K_{1}^{-1}-K_{1}, K_{2}^{-1}-K_{2}, \ldots, K_{n}^{-1}-K_{n}\right) .
$$


The necessary condition $M_{0} \geqslant 0$, eq. (7), restricts the $K s$

$$
0<K_{m} \leqslant 1, \quad m=1,2, \ldots, n .
$$

It now turns out that these restrictions on the diagonal elements in the normal form $G_{0}$ of $\mathrm{G}$ are also sufficient to ensure that $\hat{\Gamma}_{0}$, and hence $\hat{\Gamma}$, be positive semidefinite. The kernel of $\hat{T}_{0}$ is

$$
\Gamma_{0}\left(\boldsymbol{q} ; \boldsymbol{q}^{\prime}\right) \doteq \exp \left(-\frac{1}{2}{ }_{m=1}^{n}\left[K_{m}\left(q_{m}^{2}+q_{m}^{\prime 2}\right)+\frac{1}{2}\left(K_{m}^{-1}-K_{m}\right)\left(q_{m}-q_{m}^{\prime}\right)^{2}\right]\right),
$$

and it is clear that

$$
\left\langle\psi\left|\hat{\Gamma}_{0}\right| \psi\right\rangle \doteq \int \mathrm{d}^{n} q d^{n} q^{\prime} \psi(\boldsymbol{q})^{*} \Gamma_{0}\left(\boldsymbol{q} \cdot \boldsymbol{q}^{\prime}\right) \psi\left(\boldsymbol{q}^{\prime}\right) \geqslant 0,
$$

for every $\psi(\boldsymbol{q}) \in \mathrm{L}^{2}\left(\mathbb{R}^{n}\right)$ if the conditions (17) hold. Thus given that $G>0$, the inequalities (17) are the necessary and sufficient conditions for $\hat{\Gamma}_{0}$ and hence $\hat{\Gamma}$ to be positive semidefinite, or equivalently for $W(Q)$ to be a Wigner distribution.

It is possible to express the conditions (17) on $\mathrm{G}$ directly without having to bring it to its normal form. To show this and make explicit their $\mathrm{Sp}(2 n, \mathbb{R})$ invariance, we use the fact that $\beta G$ changes by a similarity transformation (13) under this group. Therefore the traces of powers of $\beta G$ are $\operatorname{Sp}(2 n, \mathrm{R})$ invariants, and can be evaluated by going to the normal form. Odd powers give vanishing trace, and for even powers we define the $\operatorname{Sp}(2 n, \mathrm{R})$ invariants

$$
S_{l}=\frac{(-1)^{l}}{2} \operatorname{Tr}(\beta G)^{2 \prime}=\underset{m=1}{£} K_{m}^{2 l}, \quad l=1,2, \ldots, n .
$$

If we now construct the nth degree polynomial equation

$$
P(y) \equiv y^{n}+c_{1} y^{n-1}+c_{2} y^{n-2}+\ldots+c_{n-1} y+c_{n}=0,
$$

where the $\mathrm{Sp}(2 n, \mathrm{R})$ invariant coefficients are given recursively by

$$
c_{m}=-\frac{1}{m}\left(S_{m}+\sum_{r=1}^{m-1} c_{r} S_{m-r} \text { I }, \quad m=1,2, \ldots, n,\right.
$$

then by Bôcher's theorem [9] the $K_{m}^{2}$ are the roots of this equation. From positivity of G follows that of each $K_{m}$, so the bound $K_{m} \leqslant 1$ is the same as $K_{m}^{2} \leqslant 1$. The necessary and sufficient conditions for all the roots of (21) to be bounded above by unity are

$$
P(1),\left(\frac{\mathrm{d} P(y)}{\mathrm{d} y}\right)_{y=1}, \ldots,\left(\frac{\mathrm{d}^{n-1} P(y)}{\mathrm{d} y^{n-1}}\right)_{y=1} \geqslant 0 .
$$

Necessity is seen by factorizing $P(y)$ as

$$
P(y)=\left(y-K_{1}^{2}\right)\left(y-K_{2}^{2}\right) \ldots\left(y-K_{n}^{2}\right),
$$

and sufficiency by expanding $P(y)$ about $y=l$. In detail the inequalities (23) are

$$
\begin{aligned}
& 1+c_{1}+c_{2}+\ldots+c_{n} \geqslant 0, \\
& n+(n-1) c_{1}+(n-2) c_{2}+\ldots+2 c_{n-2}+c_{n-1} \geqslant 0, \\
& n(n-1)+(n-1)(n-2) c_{1}+\ldots+6 c_{n-3}+2 c_{n-2} \geqslant 0, \\
& \ldots \\
& n !+(n-1) ! c_{1} \geqslant 0 .
\end{aligned}
$$


These are the $\operatorname{Sp}(2 n, \mathrm{R})$ invariant and direct expression of the conditions (17). Our results can be stated as the following

Theorem. The necessary and sufficient conditions for a gaussian phase space distribution to be a Wigner distribution, or equivalently for a gaussian kernel to represent a density matrix, are that the parameter matrix $G$ be positive definite and that the $\mathrm{Sp}(2 n, \mathrm{R})$ invariant traces of powers of $f t G$ satisfy the inequalities (25).

Quantum mechanical density matrices and optical cross-spectral densities have similar defining properties of hermiticity and positive semidefiniteness, and differ only in that the former have unit, and the latter finite, trace. Therefore our theorem applies to the optical case as well with the words "density matrix" and "Wigner distribution" replaced by "cross-spectral density" and "Wolf function" respectively.

Finally we note the nontrivial role played by the antisymmetric part of the phase matrix $K$ in kernel (1) in the context of the positive semidefiniteness of this kernel. (The symmetric part of $K$ is irrelevant for this property.) This can be seen already from the last inequality (25) which applies for all $n \geqslant 1$ :

$$
-c_{1} \leqslant n .
$$

When expressed in terms of $L, M, K$ this reads:

$$
\operatorname{Tr} L(L+M)^{-1}+\frac{1}{2} \operatorname{Tr} N^{\mathrm{T}} N \leqslant n, \quad N=-N^{\mathrm{T}}=\frac{1}{2}(L+M)^{-1 / 2}\left(K-K^{\mathrm{T}}\right)(L+M)^{-1 / 2} .
$$

It follows that the kernel ceases to be positive semidefinite if the antisymmetric part of $K$ is "large compared to $\mathrm{M}$ ". In particular if $M=0$, the positive semidefiniteness of $\hat{\Gamma}$ cannot tolerate any nonzero antisymmetric part in $K$ at all. The remaining inequalities in (25) impose further restrictions on $K-K^{\mathrm{T}}$.

We conclude with the following observation. In the general case a real phase space distribution $W(\boldsymbol{q} ; \boldsymbol{p})$ is a Wigner distribution if and only if the hermitian operator $\hat{\Gamma}$ whose configuration space kernel $\Gamma\left(\boldsymbol{q} ; q^{\prime}\right)$ computed through the inverse of the Wigner-Moyal transform

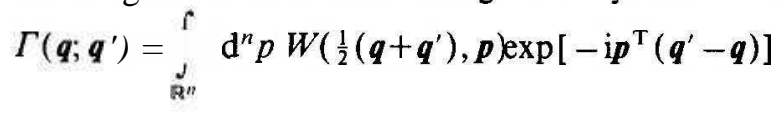

is a bona fide quantum mechanical density matrix. In practice, it is the nonnegativity requirement on $\hat{\Gamma}$ that is formidable to test, in the general case. Formally, this requirement can be rephrased in several equivalent forms. For example, $f$ will be nonnegative if and only if $\operatorname{Tr}\left(\hat{\Gamma} \hat{\Gamma}_{\psi}\right) \geqslant 0$ for every pure state density operator $\hat{\Gamma}_{\psi}$, or, equivalently, if the superposition integral of $W$ with every pure state Wigner distribution $W_{\psi}$ is nonnegative:

$$
\underset{\mathbb{R}^{2 n}}{\mathrm{~J}} \mathrm{~d}^{n} q \mathrm{~d}^{n} p W(\boldsymbol{q}, \boldsymbol{p}) W_{\psi}(\boldsymbol{q}, \boldsymbol{p}) \geqslant 0 .
$$

Another equivalent form is the KLM condition which requires the symplectic Fourier transform of $W$ to be of $h$-positive type [10]. We believe that none of these formal statements overcomes, in any definitive way, the basic difficulty involved in testing the nonnegativeness of a given A In the last KLM form, for example, we have to test the nonnegativity of an infinite sequence of matrices of increasing order constructed from the symplectic Fourier transform. What we have shown in this paper is that given a gaussian distribution in a $2 n$-dimensional phase space we can answer the question of whether it is a bona fide Wigner distribution or not by testing just $n$ simple scalar inequalities, thus giving a characterization of gaussian Wigner distributions. Clearly our conditions (25) give a much simpler algorithm than the KLM form. It has been suggested by a reviewer that it may be an interesting problem to derive our $n$ scalar conditions in (25) directly from the infinite sequence of KLM matrix conditions, for the gaussian case. We shall return to this problem elsewhere.

\section{References}

[ 1 ] R. Simon, E.C.G. Sudarshan and N. Mukunda, Phys. Rev. A 29 (1984) 3273. 
[2] R. Simon, E.C.G. Sudarshan and N. Mukunda, Phys. Rev. A 31 (1985) 2419.

[3] E. Wolf, J. Opt. Soc. Am. 68 (1978) 6.

[4] J.E. Moyal, Proc. Camb. Philos. Soc. 45 (1949) 99; E.P. Wigner, Phys. Rev. 40 (1932) 749.

[5] N.L. Balazs and B.K. Jennings, Phys. Rep. 104 (1984) 347;

M. Hillery, R.F. O'Connell, M.O. Scully and E.P. Wigner, Phys. Rep. 106 (1984) 121.

[6] E.C.G. Sudarshan, Phys. Lett. A 73 (1979) 269; Physica A 96 (1979) 315; Phys. Rev. A 23 (1981) 2802.

[7] R.G. Littlejohn, Phys. Rep. 138 (1986) 193.

[8] J. Williamson, Am. J. Math. 58 (1936) 141.

[9] L.A. Pipes and L.R. Harvill, Applied mathematics for engineers and physicists, 3rd Ed. (McGraw-Hill, New York) p. 107. [10] F.J. Narcowich and R.F. O'Connell, Phys. Rev. A 34 (1986) 1, and references therein. 